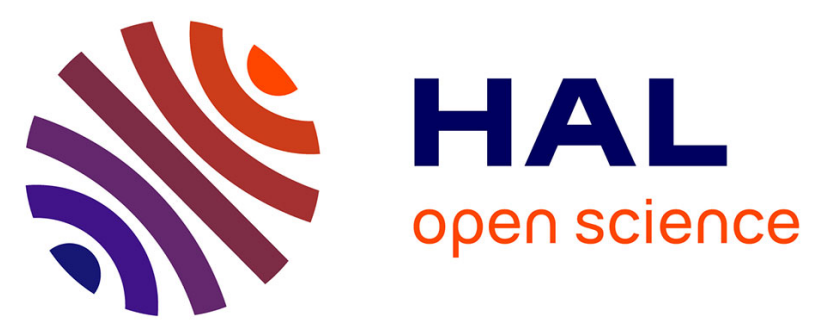

\title{
Electric field dependence on the conductivity of Kapton-HN: Integration into a 1D physical model used for the description of charge transport in dielectric materials under space environment
}

Remi Pacaud, Thierry Paulmier, Pierre Sarrailh

\section{To cite this version:}

Remi Pacaud, Thierry Paulmier, Pierre Sarrailh. Electric field dependence on the conductivity of Kapton-HN: Integration into a 1D physical model used for the description of charge transport in dielectric materials under space environment. Journal of Applied Physics, 2017, 122 (24), pp.245106 10.1063/1.5008316 . hal-01716194

\author{
HAL Id: hal-01716194 \\ https://hal.science/hal-01716194
}

Submitted on 23 Feb 2018

HAL is a multi-disciplinary open access archive for the deposit and dissemination of scientific research documents, whether they are published or not. The documents may come from teaching and research institutions in France or abroad, or from public or private research centers.
L'archive ouverte pluridisciplinaire HAL, est destinée au dépôt et à la diffusion de documents scientifiques de niveau recherche, publiés ou non, émanant des établissements d'enseignement et de recherche français ou étrangers, des laboratoires publics ou privés. 


\title{
Electric field dependence on the Conductivity of Kapton-HN: integration into a 1D Physical Model used for the description of Charge Transport in Dielectric Materials under Space Environment
}

\author{
Rémi Pacaud ${ }^{(1)}$, Thierry Paulmier ${ }^{(1)}$ and Pierre Sarrailh ${ }^{(1)}$
}

(1) Department of Physics, Instrumentation, Environment and spacE (DPhIEE), ONERA, Toulouse, 31000, France

\begin{abstract}
In this paper the development of a 1D physical conductivity model that describes charge transport in dielectric materials under space radiations with application to Kapton-HN is discussed. This model allows a good description of the charging behavior in dielectric materials. Former experiments run at ONERA on Kapton-HN showed that conductivity is temperature and electric field dependent. These results confirmed the need of implementing these dependences to our 1D model. This paper focuses on the electric field dependence. After a thorough analysis in the literature several physical theories have been found that can explain this electric field dependence on the conductivity. These are the Onsager theory and the electric field assisted de-trapping mechanisms: direct tunneling, hopping transport and the Poole-Frenkel theory. This paper is divided as follows: first the experiments that brought into evidence the electric field dependence of the conductivity in Kapton-HN are pointed out. Then the theories that could explain this dependence are described and implemented into the model. Finally, numerical and experimental conductivity results are compared and presented in the last sections.
\end{abstract}

Index Terms-Charge transport, Conductivity, Dielectrics, Direct tunneling, Electric field, Electrostatic discharges, Hopping transport, Kapton-HN, Onsager, Poole-Frenkel, Radiations, Satellites.

\section{INTRODUCTION}

In space, satellites cope with a rough radiation environment. They are constantly irradiated with high energetic and high fluxes of charged particles such as electrons and protons. Fig. 1 represents surface potentials of Teflon and Kapton as a function of irradiation time. These two materials, qualified as dielectrics, are both polymers but have very different charging behaviors. Because of charged particles impacts, they tend to get highly charged which can subsequently lead to the occurrence of electric discharges and electromagnetic disturbances that cause system degradations and satellite malfunctions. In addition, high energetic incident particles can also ionize the dielectrics. This ionization phenomenon induces changes in charge transport in the bulk material. This is due to radiation induced conductivity (RIC): the process has already been described in other papers $[1-3]$.

In order to understand charge transport in dielectric materials under space radiations, a 1D conductivity model [4] has been developed. In this paper, we specifically use the model for understanding charge transport in Kapton-HN. It had been shown in this article that the $1 \mathrm{D}$ conductivity model describes quite well the charge transport in dielectric materials under space radiations. However, this model has physical limitations since it did not fully take into account the effect of electric field on the charge transport parameters such as the charges mobility. Previous experiments [5] led by ONERA revealed that the conductivity in Kapton-HN is electric field dependent. A thorough analysis in literature brought into evidence that several theories could explain this electric field dependence: the Onsager [6 - 7] theory, direct tunneling [8], hopping transport [9] and the Poole-Frenkel [7, 8, 10] theory. We analyzed and compared those theories by implementing these in the model in order to know whether charge transport in Kapton-HN is better described.

This paper is divided as follows: section II recalls the equations of the 1D physical model as it has already been described in a previous paper [4]. Sections III and IV describe former experimental results that were carried out at ONERA [5]. These results brought into evidence the conductivity dependence with the electric field. Section V deals with the effect of the electric field on charge bulk generation when the material is irradiated with an incident electrons beam. The agreement with the Onsager theory is analyzed. Section VI deals with the effect of the electric field on the de-trapping rate of trapped charges from shallow traps. This process is named the electric field assisted de-trapping phenomena and the mechanisms behind it are direct tunneling, hopping transport and the Poole-Frenkel process. In section VII the Onsager theory and the electric field assisted de-trapping phenomena are analyzed. They are both implemented in the 1D model and numerical results are compared with experimental results. Section VIII focuses on the electric field assisted de-trapping phenomena related to deep traps. Finally, section IX gives an overall analysis of the different theories. 


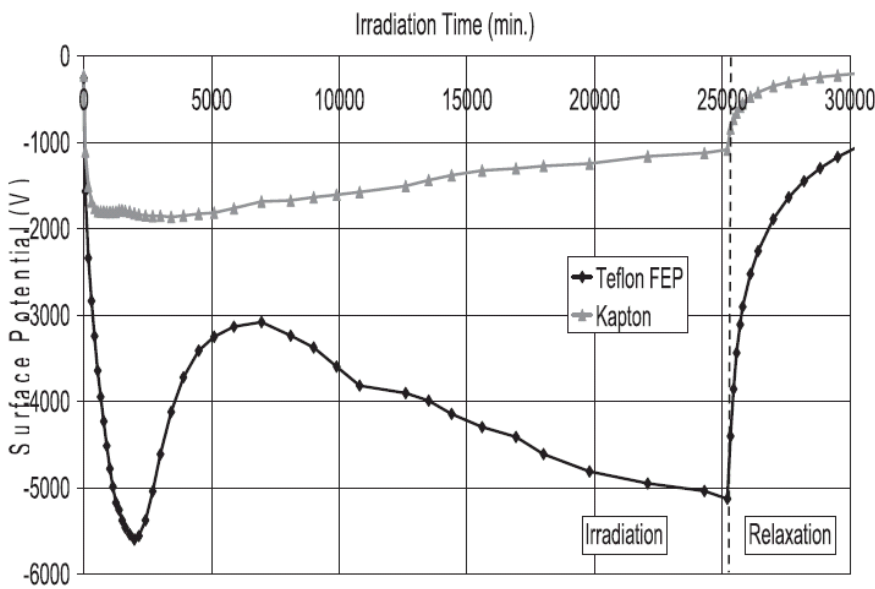

Fig. 1. Evolution of surface charging potential measured on space used polymers under GEO-like electron irradiation $(\mathrm{Kp}>5)$ - irradiation conditions: [20 keV, $\left.250 \mathrm{pA} / \mathrm{cm}^{2}\right]+\left[0-400 \mathrm{keV}, 50 \mathrm{pA} / \mathrm{cm}^{2}\right]$. From [1].

\section{DESCRIPTION OF THE CONDUCTIVITY MODEL}

In order to understand charge transport in dielectric materials under space radiations we developed a 1D conductivity model [4]. This model extends the previous OD circuit model [2].

Physical description of the $1 D$ model: when a material is irradiated with incident electrons they transfer a part of their kinetic energy to the material. This is what we usually refer to as the dose deposition profiles. This transfer of energy induces charges generation in the material bulk. Free electrons and free holes are generated into the conduction and the valence bands. These free charges diffuse in the material and move under the influence of the electric field. This is referred to as the diffusion and convection current densities. However, the material has chemical and physical defects. The material has shallow and deep trapping sites in its Fermi band where the free electrons and holes can get trapped and then de-trapped. This phenomenon is described with their trapping and detrapping characteristic time. Free electrons and free holes can also interact with each other: this is called recombination processes. In this process the electron meets a hole and returns into the valence band where it is stable and attached to an atom. Once the incident electron has transferred all its energy to the material it gets implanted in the bulk and undergoes all the previously described phenomena.

Numerical description of the $1 D$ model: the 1D model is used for the description of surface/bulk potentials and surface/bulk charge densities. The potentials are computed by the system of equations (1) with the Poisson equation and the potential boundary conditions. The bulk charge densities are computed with the system of equations (3) with the charge transport equations and the current density boundary conditions. In this system we compute the time and space evolution of the bulk charge densities that are used to compute the surface potential of the irradiated materials.

Poisson equation and boundary conditions:

$V(x)$ is the depth-dependent potential (Volt) which is the solution of the Poisson equation.

$$
\left\{\begin{array}{l}
-\Delta V(x)=\frac{\rho(x)}{\varepsilon} \\
\left.\nabla V(x)\right|_{x=0}-\frac{\varepsilon_{0}}{\varepsilon} \frac{V(0)}{L}=0 \\
V(d)=0
\end{array}\right.
$$

Where $\varepsilon$ and $\varepsilon_{0}$ are respectively the dielectric permittivity $\left(F . m^{-1}\right)$ and the vacuum permittivity $\left(F . m^{-1}\right) . L(m)$ is the distance between the front surface of the dielectric and the first surface electrically grounded. $d$ is the material thickness $(m) . \rho(x)$ is the depth-dependent total charge density $\left(C . m^{-3}\right)$ :

$$
\frac{\rho(x)}{e}=-n(x)+p(x)-n_{t}(x)+p_{t}(x)
$$

Where $e$ is the electron charge (Coulomb). $n, p, n_{t}$ and $p_{t}$ are respectively the free electron density $\left(\mathrm{m}^{-3}\right)$, the free hole density $\left(\mathrm{m}^{-3}\right)$, the trapped electron density $\left(\mathrm{m}^{-3}\right)$ and the trapped hole density $\left(\mathrm{m}^{-3}\right)$.

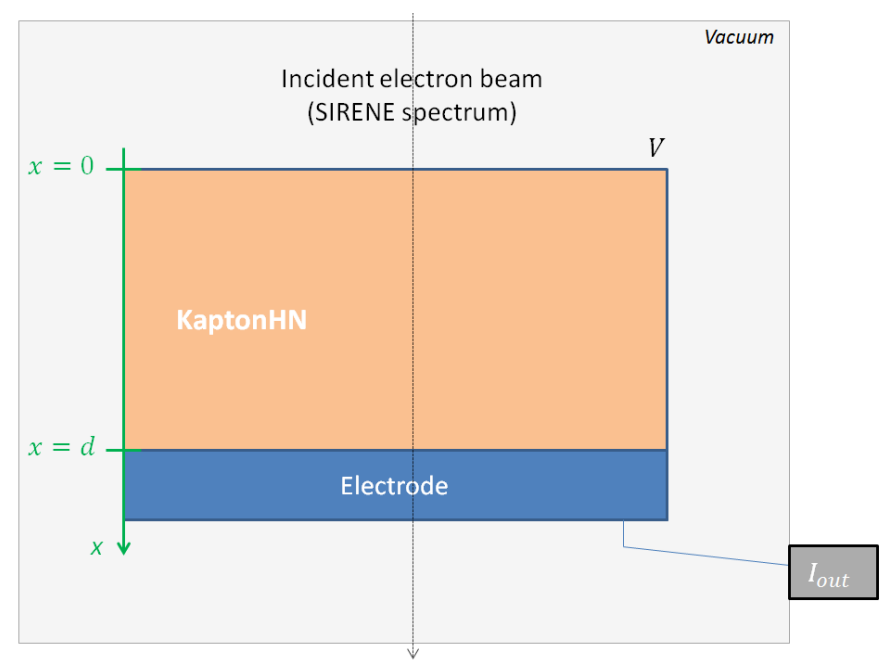

Fig. 2. One dimensional (1D) representation of a Kapton-HN sample coated on its back face with a conductive material and in direct contact with vacuum on its front face. $x=0$ represents the irradiated dielectric surface and $x=d$ is the junction between the dielectric and the metal. $V$ is the surface potential.

Description of the time/space evolution of bulk charge densities:

In order to assess the potential we need to assess the bulk charge densities as a function of irradiation time and space. System (3) describes the time and space evolution of charge densities with the current density boundary conditions of fig. 2. 


$$
\left\{\begin{array}{l}
\frac{\partial n(x, t)}{d t}=g(x, t)-\frac{\nabla \cdot j_{0}}{e}-\nabla \cdot J_{n}-R_{1}-R_{2}-\frac{n}{\tau_{n}}+\frac{n_{t}}{\tau_{n t}} \\
\frac{\partial n_{t}(x, t)}{d t}=-R_{3}+\frac{n}{\tau_{n}}-\frac{n_{t}}{\tau_{n t}} \\
\frac{\partial p(x, t)}{d t}=g(x, t)+\nabla \cdot J_{p}-R_{3}-R_{2}-\frac{p}{\tau_{p}}+\frac{p_{t}}{\tau_{p_{t}}} \\
\frac{\partial p_{t}(x, t)}{d t}=-R_{1}+\frac{p}{\tau_{p}}-\frac{p_{t}}{\tau_{p_{t}}} \\
\left.J_{n}(x, t)\right|_{x=0}=0 \\
\left.\left(\nabla \cdot J_{n}\right)\right|_{x=d}=0 \\
\left.J_{p}(x, t)\right|_{x=0}=0 \\
\left.\left(\nabla \cdot J_{p}\right)\right|_{x=d}=0
\end{array}\right.
$$

Where $g(x, t)$ is the generation rate of free electron-hole pairs $\left(m^{-3} \cdot s^{-1}\right) \cdot \nabla \cdot j_{0}$ is the incident electron implantation current in the material $\left(C \cdot m^{-3} . s^{-1}\right) . \tau_{n}, \tau_{n_{t}}, \tau_{p}$ and $\tau_{p_{t}}$ are respectively the trapping characteristic time and the de-trapping characteristic time for electrons and holes (s). Boundary conditions are chosen as follows: 1) the electron and hole currents are zero at the dielectric/vacuum junction and 2) electrons and holes can freely move at the dielectric/metal junction.

\section{Conduction currents:}

$J_{n}$ and $J_{p}$ are the electron current density $\left(m^{-2} \cdot s^{-l}\right)$ and the hole current density $\left(\mathrm{m}^{-2} \cdot \mathrm{s}^{-1}\right)$ that are the sum of the convection and the diffusion terms.

$$
\left\{\begin{array}{l}
J_{n}=J_{n}^{\text {Convection }}+J_{n}^{\text {Diffusion }}=n \mu_{n} E+D_{n} \nabla n \\
J_{p}=J_{p}^{\text {Convection }}+J_{p}^{\text {Diffusion }}=p \mu_{p} E+D_{p} \nabla p
\end{array}\right.
$$

Where $\mu_{n}$ and $\mu_{p}$ are the free electrons and free holes mobility. $D_{n}$ and $D_{p}$ are the free electron and free holes diffusion coefficients. $E$ is the electric field $\left(V . m^{-l}\right)$ and is time and space dependent.

\section{Recombination terms:}

$R_{l}$ is the recombination term $\left(\mathrm{m}^{-3} \cdot \mathrm{s}^{-1}\right)$ between free electrons and trapped holes and $\alpha_{1}$ is its corresponding recombination coefficient $\left(m^{3} \cdot s\right) . R_{2}$ is the recombination term $\left(m^{-3} \cdot s^{-1}\right)$ between free electrons and free holes and $\alpha_{2}$ is its corresponding recombination coefficient $\left(m^{3} . s\right) . R_{3}$ is the recombination term $\left(\mathrm{m}^{-3} \cdot \mathrm{s}^{-1}\right)$ between trapped electrons and free holes and $\alpha_{3}$ is its corresponding recombination coefficient $\left(\mathrm{m}^{3} . s\right)$.

$$
\left\{\begin{array}{l}
R_{1}=\alpha_{1} n p_{t} \\
R_{2}=\alpha_{2} n p \\
R_{3}=\alpha_{3} n_{t} p
\end{array}\right.
$$

As discussed in a previous paper [4] the 1D model gives a good description of charge transport in dielectric materials. But as stated in section I it has limitations because it does not fully take into account electric field effects on charge transport. This drawback can lead to significant deviations in regard of the experimental values. In the next section we present the experiments that brought into evidence the electric field dependence on Kapton-HN electric conductivity.

\section{EXPERIMENTS}

In order to study charge transport under space radiations three different and specific experimental configurations have been set up at ONERA. In the first part of this section we describe each configuration and in the second part we analyze the purpose behind each of these configurations.

$1^{\text {st }}$ configuration: In this configuration the sample is irradiated for 2 hours with the SIRENE spectrum [11] and relaxed for 3 hours. Fig. 3 represents the integrated current density $J_{i}$ of the incident electrons as a function of the energy. The SIRENE spectrum reproduces the geostationary spectrum with index $K_{p}>5$ which allows irradiating the sample in realistic conditions. Fig. 2 schematically represents the irradiation of a sample of Kapton-HN of $127 \mu \mathrm{m}$ under the SIRENE spectrum. The vacuum level in the chamber is $10^{-6} \mathrm{hPa}$ and the material is grounded on its back face with a conductive electrode. In this experiment the surface potential is monitored with a Kelvin probe. This experiment will be referred to as the $I^{\text {st }}$ configuration.

$2^{\text {nd }}$ configuration: In this configuration the sample is irradiated for 30 seconds with a mono-energetic electron beam of 400 $\mathrm{keV}$ and a flux of $6 \mathrm{pA} / \mathrm{cm}^{2}$. Irradiation is followed by 30 minutes of relaxation. Fig. 4 schematically represents the irradiation of a Kapton-HN sample of $50 \mu \mathrm{m}$ under the monoenergetic electron beam. The vacuum level in the chamber is $10^{-6} \mathrm{hPa}$ and the material is coated on its front and back faces with a conductive electrode. A negative voltage is applied on electrode 1 while electrode 2 is grounded. As a result, one can apply a constant (time independent) electric field on the sample and dissociate effect of dose and effect of electric field on radiation induced conductivity. This experiment will be referred to as the $2^{\text {nd }}$ configuration.

$3^{\text {rd }}$ configuration: In this configuration the sample is irradiated for 30 minutes with a mono-energetic electron beam of 400 $\mathrm{keV}$ and a flux of $4 \mathrm{pA} / \mathrm{cm}^{2}$. Irradiation is followed by 2 hours of relaxation. The geometry is exactly the same than in the $2^{\text {nd }}$ configuration (fig. 4). However, the sample thickness here is $25 \mu \mathrm{m}$. This experiment will be referred to as the $3^{\text {rd }}$ configuration. 
Each configuration has a specific purpose. The $1^{\text {st }}$ configuration is used for understanding the influence of high dose rates and electron implantation on the dielectric RIC (as met in a space environment). In order to gauge it, a Kelvin Probe is used for surface potential measurements and a picoammeter for current densities measurements. The implanted electrons lead to a space charge region which induces an internal electric field. This electric field steers the RIC which makes this configuration the most complicated configuration from all three. Subsequently, the $1^{\text {st }}$ configuration is used as a mean to fully validate the $1 \mathrm{D}$ model (section VIII).

$2^{\text {nd }}$ and $3^{\text {rd }}$ configurations have simpler irradiation and geometry conditions than the $1^{\text {st }}$ configuration. These configurations are used for understanding the influence of the electric field on the RIC (section IV - VII). Due to low fluxes and high energetic incident electrons, dose rates and electron implantation are weak. Subsequently, it does not lead (like in the $1^{\text {st }}$ configuration) to a bulk space charge and a space inhomogeneous internal electric field that steer the RIC. Here, the only existing electric field is the constant macroscopic electric field that is applied on the material with the electrodes. The electric field has been applied long before the irradiation started in order to prevent any effect due to the polarization of the dielectric on the conductivity. Working under these conditions thus enables to truly see the influence of the electric field on the RIC. Moreover, as we will see in the next section, it justifies neglecting the electron deposition term, the recombination terms and the convection/diffusion current densities in system (3). This simplification allows a better understanding of the electric field influence on charge transport. In the $2^{\text {nd }}$ configuration short terms effects of dose deposition are studied on the RIC. In the $3^{\text {rd }}$ configuration long term effects of dose deposition are studied on the RIC.

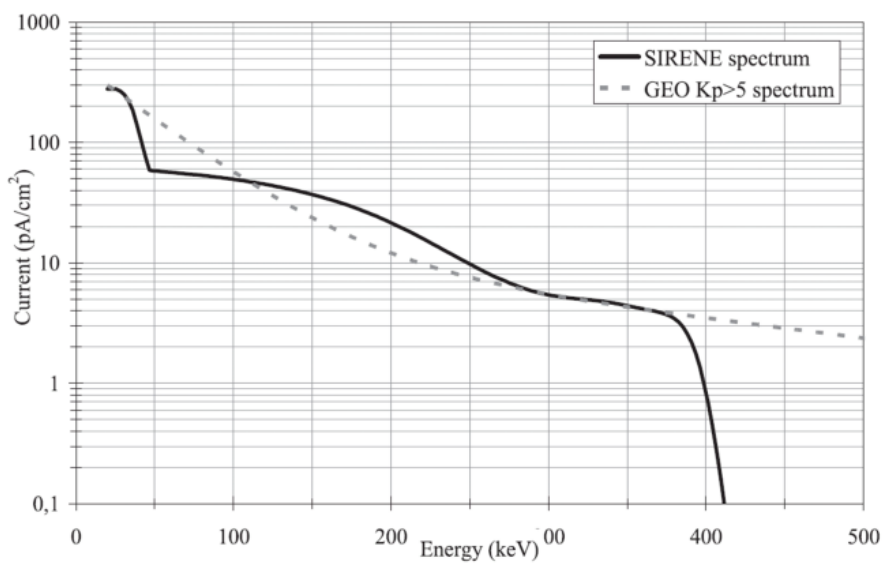

Fig. 3. SIRENE standard spectrum and reference Kp $>5$ spectrum. From [11].

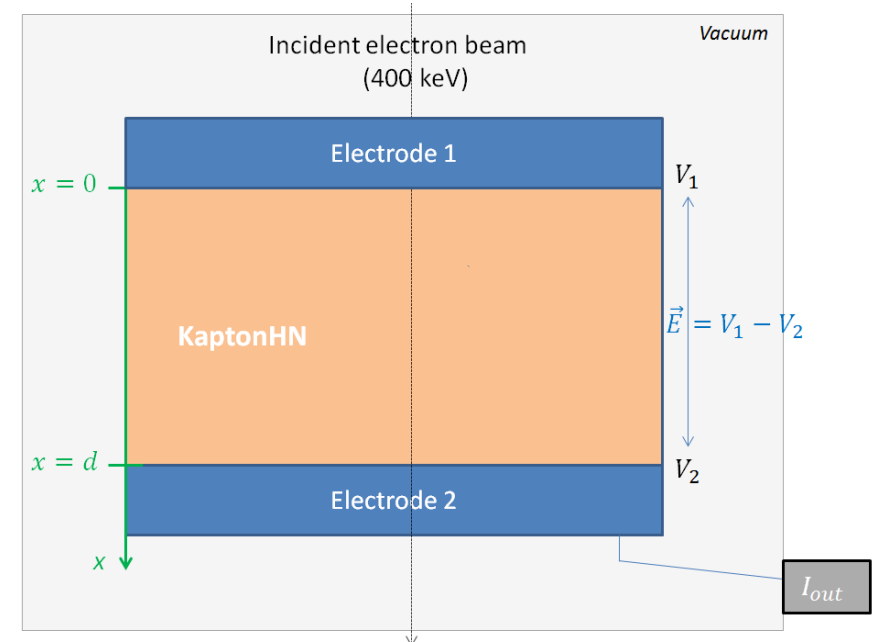

Fig. 4. One dimensional (1D) representation of a Kapton-HN sample coated on its front face and back face with a conductive material. $\mathrm{x}=0$ and $\mathrm{x}=\mathrm{d}$ are both junctions between the dielectric and the electrodes. The sample is irradiated with a $400 \mathrm{keV}$ electron beam that goes through the sample. There is no electron implantation. A voltage difference is applied between the electrodes leading to a constant macroscopic electric field during the test.

\section{PHENOMENOLOGY}

In this section conductivity measurements are presented with the $2^{\text {nd }}$ and $3^{\text {rd }}$ configurations described in section III. It allows using a simplified formula for the conductivity in order to analyze which term present in system (3) is electric field dependent.

Fig. 5 displays conductivity measurements $\left(2^{\text {nd }}\right.$ configuration, section III) as a function of irradiation time for 6 different potentials on electrode $1:-100 \mathrm{~V},-200 \mathrm{~V},-400 \mathrm{~V},-600 \mathrm{~V},-800 \mathrm{~V}$ and $-1000 \mathrm{~V}$. The typical electric field value for an electrical breakdown for a sample of Kapton-HN of $50 \mu \mathrm{m}$ is $3 \times$ $10^{8} \mathrm{~V} \cdot \mathrm{m}^{-1}$. The highest electric field we work with in this

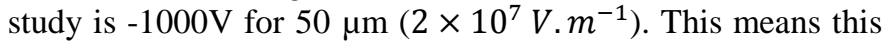
is one order of magnitude below the threshold before an electrical breakdown occurs. Conductivity $\sigma$ is computed from current density measurements:

$\sigma=\frac{J_{\exp }}{E}$

$\sigma\left(\Omega^{-1} m^{-1}\right)$ is the computed conductivity, $J_{\text {exp }}\left(A . m^{-2}\right)$ is the measured current density and $E\left(V . m^{-1}\right)$ the applied electric field. Results of fig. 5 clearly show that conductivity is electric field dependent. For each imposed electric field, conductivity shows four phases: a transient phase where conductivity rapidly increases (first 3 seconds after irradiation began), a slow increase of conductivity $\left(3^{\text {rd }}\right.$ to $30^{\text {th }}$ second after irradiation began), a drop of conductivity when irradiation is stopped and a slow decrease of conductivity during the rest of the relaxation phase. During the first transient mode the conductivity is directly proportional to the number of free electrons and free holes generated in the bulk. After few seconds of irradiation charges start to get trapped and conductivity stops increasing as fast as during the transient phase. Since the number of trapped charges increases, the detrapping rate of these charges increases as well. As a result, 
the generation rate of free electrons and free holes added to the de-trapping rate of these charges slightly overcomes their trapping rate: the conductivity slowly increases. When irradiation is stopped the generation rate of free electrons and free holes stops. In addition, free charges still get quickly trapped which results in a quick drop of the conductivity. After few seconds the conductivity slowly decreases due to a slow de-trapping of charges that leads to recombination processes. The presence of the RIC after irradiation is stopped is called delayed RIC (DRIC) and has already been described in another paper [3].

In order to decrease the measured signal noise, the conductivity was averaged over the phase of slow increase of the conductivity during irradiation (between the $10^{\text {th }}$ and $30^{\text {th }}$ seconds) for each electric field and plotted in fig. 6. Fig. 6 represents the natural logarithm of the averaged conductivity as a function of the square root of the electric field. It shows a straight dependence with the square root of the electric field.

Fig. 7 displays conductivity measurements (using the $3^{\text {rd }}$ configuration) as a function of irradiation time for 4 different voltages on electrode 1: $-400 \mathrm{~V},-800 \mathrm{~V},-1000 \mathrm{~V}$ and $-2000 \mathrm{~V}$. The conductivity is computed from current density measurements using equation (6). Results of fig. 7 show that conductivity is electric field dependent. For each electric field the behavior of the conductivity as a function of irradiation time is globally the same as in fig. 5. However, since irradiation time is longer in the $3^{\text {rd }}$ configuration than in the $2^{\text {nd }}$ configuration, the total amount of dose deposition is greater. The number of charges filling the trapping sites is greater which leads to a significant increase of the de-trapping rate of charges: the increase of the RIC during irradiation is greater in the $3^{\text {rd }}$ configuration than in the $2^{\text {nd }}$ configuration. During the relaxation phase the de-trapping rate of charges is higher in the $3^{\text {rd }}$ configuration than in the $2^{\text {nd }}$ configuration. The number of free charges, thus the recombination rate and therefore the decrease of the DRIC in the $3^{\text {rd }}$ configuration are greater than in the $2^{\text {nd }}$ configuration.

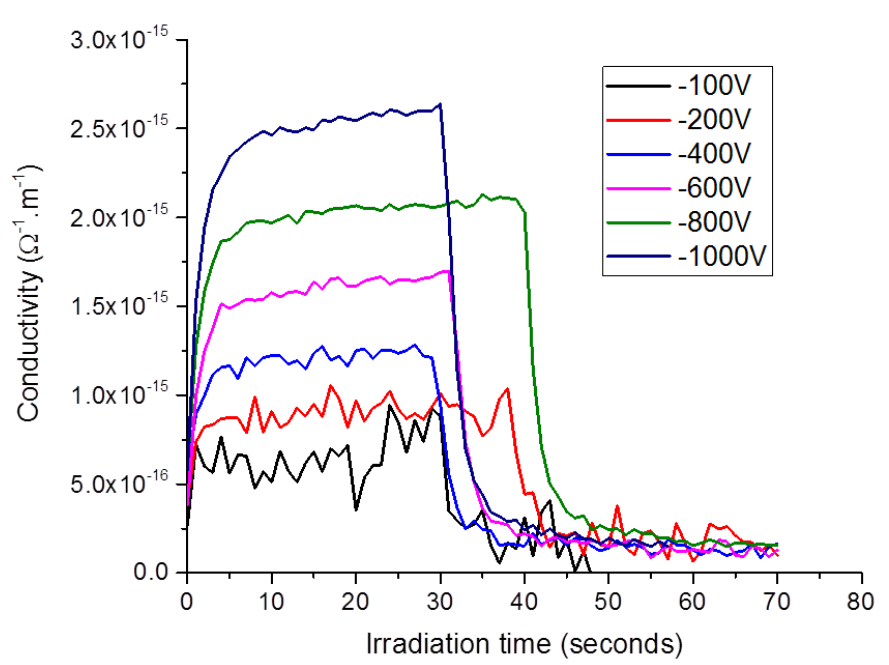

Fig. 5. Conductivity measurements for 6 different voltages. The experimental configuration refers to the one from fig. 4 . The material is a $50 \mu \mathrm{m}$ thick Kapton-HN sample. During the first phase the dielectric is irradiated for 30 seconds. In the second phase the irradiation was stopped and the dielectric is relaxed for several minutes, but the only first 30 seconds are displayed.

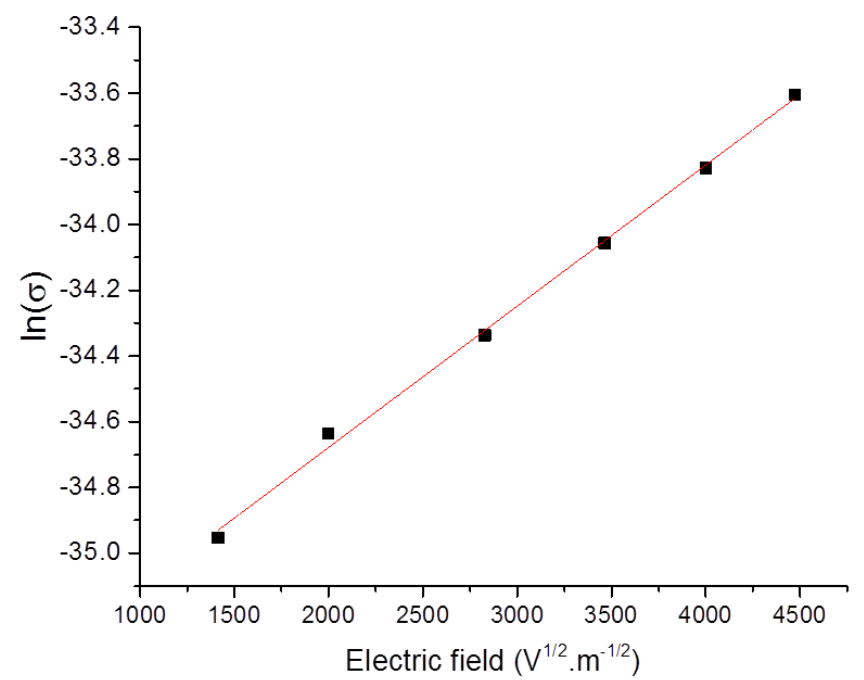

Fig. 6. Natural logarithm of the conductivity as a function of the square root of the electric field. Conductivities are those from fig. 5. They were averaged over the plateau for each electric field.

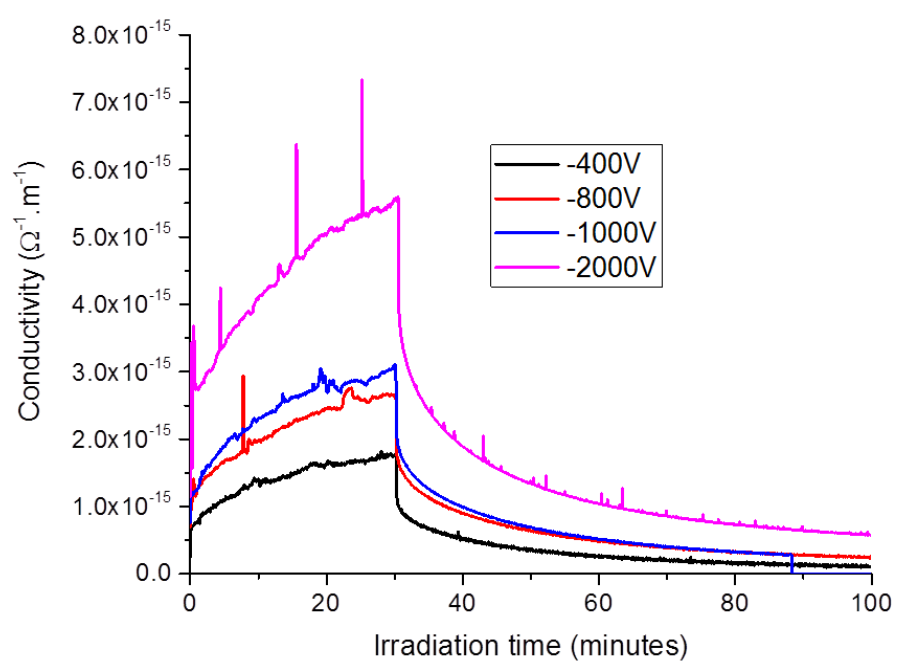

Fig. 7. Conductivity measurements for 4 different applied electric fields. The experimental configuration refers to the one from fig. 4 . The material is a 25 $\mu \mathrm{m}$ thick Kapton-HN sample. During the first phase the dielectric is irradiated for 35 minutes. In the second phase the irradiation was stopped and the dielectric is relaxed for over an hour.

In order to define which term of system (3) is electric field dependent a simplified formula for the conductivity has been computed. As conductivity is electric field dependent (fig. 5, fig. 6 and fig. 7), we assume that the terms present in such a formula are likely to be electric field dependent.

Let us now refer back to system (3) from section II. With a configuration such as the $2^{\text {nd }}$ configuration the complexity of the problem is reduced. In system (3) several terms of this system can be neglected:

1) There is no electron deposition in the sample, thus: $\nabla \cdot j_{0}=0$.

2) Due to low electron fluxes and with short irradiation times (less than a minute), one can neglect the recombination terms with respect to the others (electron implantation, generation 
rate of free charges, trapping and de-trapping terms and conduction currents).

3) Because a constant macroscopic electric field is applied, there is no electron implantation and the deposited dose profile is uniform in the material bulk, one can assume that everything is uniform in the volume. Therefore, the gradients are negligible: $\nabla \cdot J_{n}=\nabla \cdot J_{p} \approx 0$. With these 3 hypotheses, system (3) changes into system (6):

$$
\left\{\begin{array}{l}
\frac{\partial n(x, t)}{d t}=g(x, t)-\frac{n}{\tau_{n}}+\frac{n_{t}}{\tau_{n_{t}}} \\
\frac{\partial n_{t}(x, t)}{d t}=\frac{n}{\tau_{n}}-\frac{n_{t}}{\tau_{n_{t}}} \\
\frac{\partial p(x, t)}{d t}=g(x, t)-\frac{p}{\tau_{p}}+\frac{p_{t}}{\tau_{p_{t}}} \\
\frac{\partial p_{t}(x, t)}{d t}=\frac{p}{\tau_{p}}-\frac{p_{t}}{\tau_{p_{t}}}
\end{array}\right.
$$

The system (6) has an analytical solution for $n(x, t)$ and $p(x, t)$. The general solution for $n(x, t)$ (which is the same as for $p(x$, $t)$ ) is shown by equation (7).

$n(x, t)=\frac{g \tau_{n}}{\left(\frac{\tau_{n}}{\tau_{n_{t}}}+1\right)^{2}}\left(1-e^{-\left(\frac{1}{\tau_{n}}+\frac{1}{\tau_{n_{t}}}\right) t}\right)+\frac{g}{\left(\frac{\tau_{n_{t}}}{\tau_{n}}+1\right)} t$

Where $g=g(x, t)$.

If we assume that the characteristic trapping time of electrons and holes is much lower than their characteristic de-trapping time, it means that: $\tau_{n} \ll \tau_{n_{t}}$ and $\tau_{p} \ll \tau_{p_{t}}$. Equation (7) can thus be simplified into equation (8).

$n(x, t) \approx g \tau_{n}\left(1-e^{-\frac{t}{\tau_{n}}}\right)+g \frac{\tau_{n}}{\tau_{n_{t}}} t \approx g \tau_{n}\left(1-e^{-\frac{t}{\tau_{n}}}\right)$

Conductivity is computed by equation (9).

$\sigma=e\left(n \times \mu_{n}+p \times \mu_{p}\right)$

Using equation (8), we can get a more simplified formula for the conductivity:

$\sigma \approx e g\left(\tau_{n} \mu_{n}\left(1-e^{-\frac{t}{\tau_{n}}}\right)+\tau_{p} \mu_{p}\left(1-e^{-\frac{t}{\tau_{p}}}\right)\right)$

As discussed before, fig. 6 and fig. 7 show that conductivity is electric field dependent. This means that in equation (10), either $g(x, t), \mu_{n}, \mu_{p}$ or all of them are electric field dependent. After a thorough analysis in the literature it appeared that several theories are likely to explain this dependence. The first envisaged theory is the Onsager theory. It considers that the generation rate of electron-hole pairs $g(x, t)$ is temperature and electric field dependent: $g(x, t)=$ $g(x, t, T, E)$. It is discussed in section V. The other theories have an influence on the transport parameters and are referred to as the electric field assisted de-trapping processes. They are the direct tunneling, the hopping transport and the PooleFrenkel theory. They consider that the de-trapping rate of electrons and holes from shallow trapping sites is electric field dependent. In the literature this phenomenon has been described with the mean mobility of free charges being electric field dependent: $\mu_{n}=\mu_{n}(E)$ and $\mu_{p}=\mu_{p}(E)$. It is discussed in section VI. For deep trapping sites only the Poole-Frenkel effect occurs. It steers the de-trapping rate of electrons and holes from deep traps and it is discussed in section VIII.

Nota Bene: In order to obtain equation (10), the hypothesis of short irradiation times has been made. This hypothesis is a way to simplify system (3) and bring into evidence which term is electric field dependent. Therefore, these terms are always electric field dependent, even for long irradiation times.

\section{ONSAGER MODEL}

By definition the Onsager theory considers that the generation rate of electron-hole pairs $g(x, t)$ is temperature and electric field dependent: $g(x, t)=g(x, t, T, E)$. This dependence is controlled by a factor, named $\varphi$ [6], that gives the probability that a thermalized electron escapes recombination with its parent ion for a specific electric field and a specific temperature. Subsequently, one must add this factor to the generation rate of electrons and holes as follows: $g(x, t, T, E)=\varphi(T, E) \times g(x, t)$. The corresponding equation for $\varphi$ is the following:

$\varphi=\varphi_{0} \frac{k T}{e E r_{0}} e^{\frac{-e^{2}}{4 \pi \kappa \varepsilon_{0} k T r_{0}}} e^{\frac{-e E r_{0}}{k T}}$
$\sum_{m=0}^{\infty} \frac{A^{m}}{m !} \sum_{n=0}^{\infty} \sum_{l=m+n+1}^{\infty}\left(\frac{e E r_{0}}{k T}\right)^{l} \frac{1}{l !}$

Where $\varphi_{0}$ is the efficiency of production of thermalized ion pairs per absorbed electron. $r_{0}$ is the thermalization radius. It corresponds to the distance between the thermalized electron and its parent ion. In [7] Tyutnev has arranged the previous equation and found an analytical equation:

$\varphi=\varphi_{0} \exp \left(\frac{e^{2}}{4 \pi \varepsilon \varepsilon_{0} r_{0}} \frac{\exp \left(-\frac{e E r_{0}}{k T}\right)-1}{\frac{e E r_{0}}{k T}}\right)$

Equations (11) and (12) were compared in fig. 8 (left axis) plotted as a function of the electric field for two different $r_{0}$ and for a temperature of $300 \mathrm{~K}$. In fig. 8 (right axis) the relative change between equations (11) and (12) is plotted in percentage for $r_{0}=14 \mathrm{~nm}$. Relative change is very low (maximum 3.5\% change around $10^{7} \mathrm{~V} / \mathrm{m}$ ), which shows that both equations give almost identical results. But it appeared that the evaluation of equation (11) is much more time consuming than equation (12). Therefore, in the next sections, the Onsager theory will be described with equation (12). 


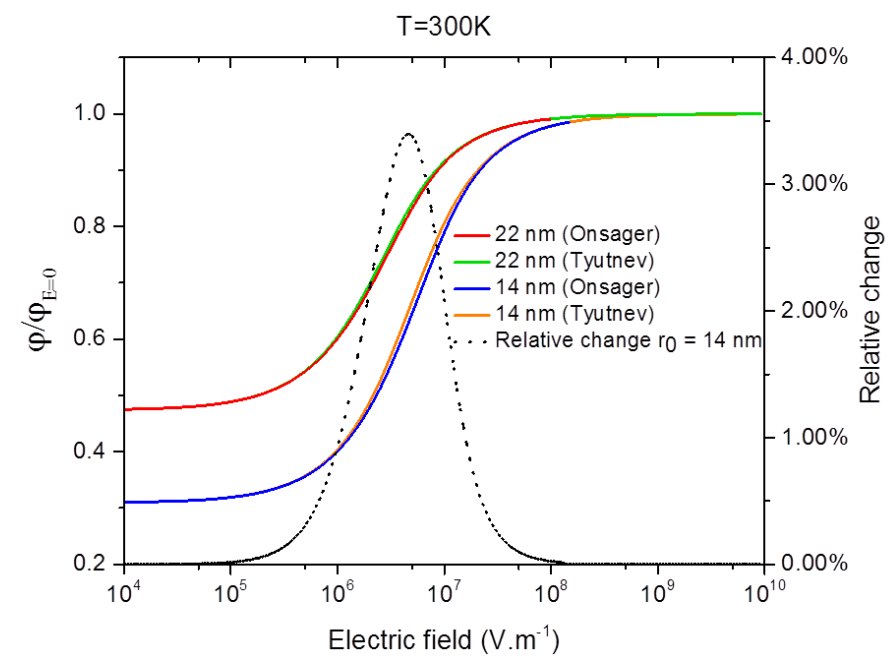

Fig. 8. Comparison of the Onsager equation and the Tyutnev modified equation of Onsager for a temperature of $300 \mathrm{~K}$ as a function of the electric field with dependence on the thermalization radius.

According to the definition of the Onsager theory the generation rate of electron-hole pairs is directly modified by the $\varphi$ factor. Therefore, one must add the $\varphi$ factor to equation (10) as follows:

$\sigma \approx \operatorname{e\varphi g}(x, t)\left(\tau_{n} \mu_{n}\left(1-e^{-\frac{t}{\tau_{n}}}\right)+\tau_{p} \mu_{p}\left(1-e^{-\frac{t}{\tau_{p}}}\right)\right)$

\section{Parameters determination:}

The objective is to study whether the Onsager theory can explain the electric field dependence on the conductivity (fig. 6 and fig. 7). Assuming that the mobility of charges does not depend on the electric field then $\sigma$ must follow the same dependency as $\varphi: \partial \sigma / \partial E=\partial \varphi / \partial E$. In fig. 9 we compare the conductivities from fig. 6 and fig. 7 with equation (12). Conductivities from fig. 6 and fig. 7 as well as equation (12) are plotted as a function of electric field. The conductivities from fig. 7 were averaged during the first 30 seconds after irradiation started. All conductivities were then normalized to the conductivity that corresponds to $E=1,6 \times 10^{7} \mathrm{~V} / \mathrm{m}$. Equation (12) was also normalized for the same electric field. We tried to fit the experimental data from fig. 6 and fig. 7 with equation (12) with several thermalization radius: $r_{0}=7 \mathrm{~nm}$, $r_{0}=15 \mathrm{~nm}$ and $r_{0}=30 \mathrm{~nm}$. The best fit corresponds to $r_{0}=7 \mathrm{~nm}$. For low electric fields (roughly lower than $5 \times 10^{6}$ $\mathrm{V} / \mathrm{m}$ ) the conductivity follows the same law as the Onsager theory for $r_{0}=7 \mathrm{~nm}$. However, for higher electric fields, it is clear that conductivity does not obey to the Onsager theory. In the next section we talk about the electric field assisted detrapping mechanisms that may better explain the conductivity behavior for high electric fields.

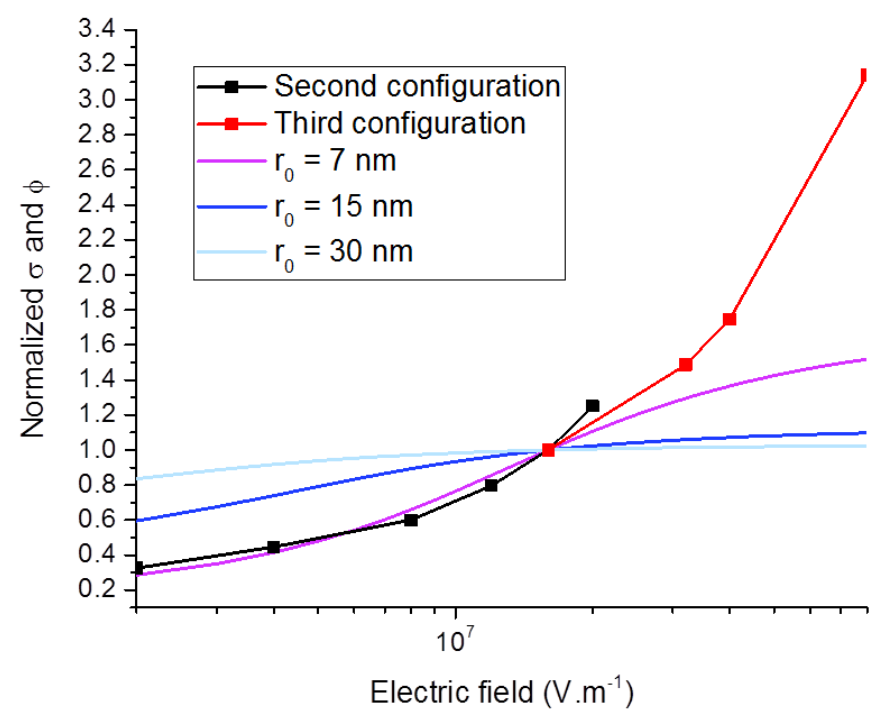

Fig. 9. Comparison between the measured conductivities from fig. 6 and fig. 7 with the Tyutnev modified equation (equation (12)) of Onsager with three different thermalization radius: $r_{0}=7 \mathrm{~nm}, r_{0}=15 \mathrm{~nm}$ and $r_{0}=30 \mathrm{~nm}$.

\section{ELECTRIC FIELD ASSISTED DE-TRAPPING PROCESSES RELATED TO SHALLOW TRAPS}

In this section, data from fig. 7 have been analyzed in order to study any possible influence of electric field assisted detrapping processes. In order to exclude the Onsager process the analysis focuses on the relaxation phase. During the relaxation phase the irradiation is stopped, thus $g(x, t, T, E)=$ 0 . In this phase, the electric field only acts on the de-trapping rate of charges from shallow trapping sites.

\section{Definition:}

In literature it is stated that direct tunneling, hopping transport or the Poole-Frenkel process act on charge de-trapping (especially for shallow trapping sites). Under the influence of a high external electric field, the de-trapping rate of electrons and holes from shallow traps increases and leads to an increase of the conductivity. In the meantime, free electrons and free holes are still being quickly trapped in the Anderson states [12] (shallow traps). An equilibrium appears between de-trapping (steered by the electric field) and trapping of charges, which thus increases the mean mobility of free electrons and free holes. In [7, 13] Tyutnev suggested that this equilibrium can be computed by implementing an electric field dependence on the mean mobility of free electrons and free holes:

$\left\{\begin{array}{l}\ln \left(\mu_{n}\right)=\beta \times E^{1 / 2}+\ln \left(\mu_{n_{0}}\right) \\ \ln \left(\mu_{p}\right)=\beta \times E^{1 / 2}+\ln \left(\mu_{p_{0}}\right)\end{array}\right.$

Where $\mu_{n_{0}}$ and $\mu_{p_{0}}$ are the mobility at zero electric field. $\beta \times E^{1 / 2}$ corresponds to a lowering effect on the Coulomb barrier. $\beta$ gathers all three processes: direct tunneling, hopping transport and Poole-Frenkel. If we assume that the mean mobility of free electrons and free holes is steered by the 
electric field then the conductivity from equation (9) can be written as:

$\ln (\sigma)=\beta \times E^{1 / 2}+\ln \left(\sigma_{0}\right)$

Where $\sigma_{0}$ is the conductivity at zero external electric field on the material.

\section{Parameters determination:}

$\beta$ is computed by fitting experimental results (fig. 10) with equation (15). In fig. 7, during the relaxation phase, we selected three different periods of time for a duration of 60 seconds each: $32^{\text {th }}$ to $33^{\text {th }}$ minute, $40^{\text {th }}$ to $41^{\text {th }}$ minute and $60^{\text {th }}$ to $61^{\text {th }}$. Conductivity was then averaged over these ranges of time for each electric field and displayed on fig. 10. On this figure the natural logarithm of conductivity $\sigma$ is represented as a function of $E^{1 / 2}$ : the results are straight lines. Therefore, it strongly suggests that the conductivity as well as the electron and hole mobility is steered by the electric field assisted detrapping processes (direct tunneling, hopping transport and Poole-Frenkel). A linear regression is done on fig. 10 represented by the black straight lines. The slopes of these straight lines correspond to the value of $\beta$ and yielded to $\beta=3 \times 10^{-4} \mathrm{~m}^{1 / 2} \cdot V^{1 / 2}$.

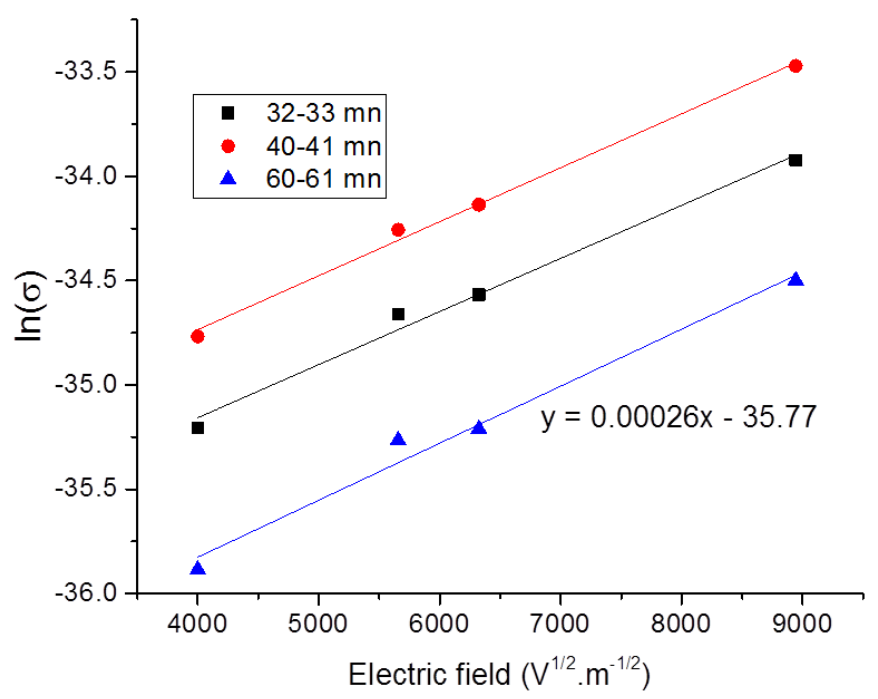

Fig. 10. Plot of the natural logarithm of conductivity as a function of the square root of the electric field. Conductivity has been averaged for each electric field over three different range of time of 60 seconds duration each: $32^{\text {th }}$ to $33^{\text {th }}$ minute, $40^{\text {th }}$ to $41^{\text {th }}$ minute and $60^{\text {th }}$ to $61^{\text {th }}$ minute after relaxation began.

\section{ONSAGER AND ELECTRIC FIELD ASSISTED DE- TRAPPING PROCESSES RELATED TO SHALLOW TRAPS}

In the two previous sections we described the Onsager mechanism and the electric field assisted de-trapping processes related to shallow traps. In this section we implement in the 1D model [4] both mechanisms and see, when they are combined, whether they can explain the experimental results. Firstly, we use the data from fig. 5 and try to reproduce these using only the theory of the electric field assisted de-trapping processes (equation (14)) related to shallow traps. In this first set of simulations, the Onsager mechanism is neglected. Results are displayed on fig. 11 and yield very good agreements. In fig. 12 we then tried to reproduce the same behavior using both mechanisms (the Onsager mechanism is taken into account). The results are only slightly better from those displayed in fig. 11. It suggests that the Onsager mechanism is quite weak for Kapton-HN. The electric field assisted de-trapping phenomena are responsible of the conductivity behaviors.

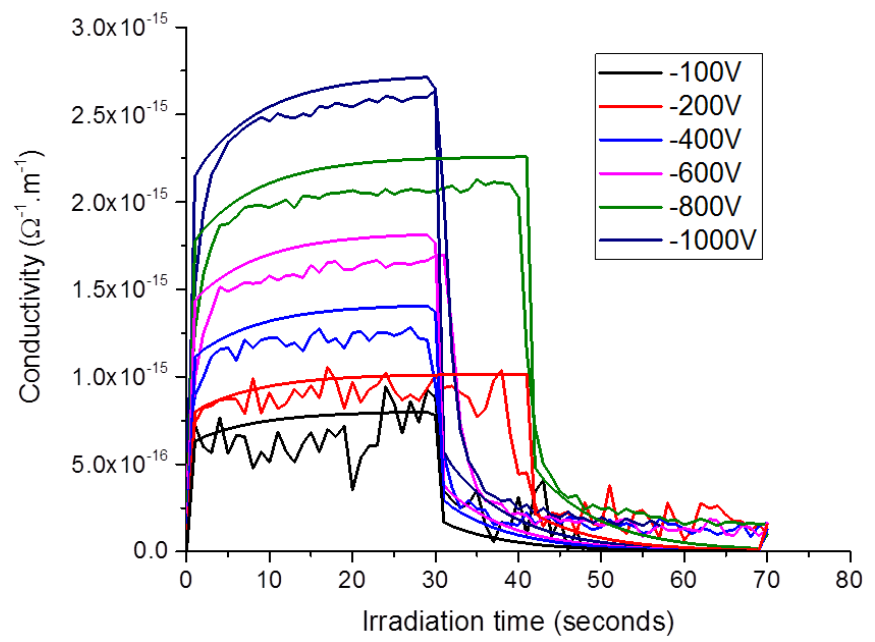

Fig. 11. Results are those from fig. 5. Fits are made using the theory related to the electric field assisted de-trapping phenomena.

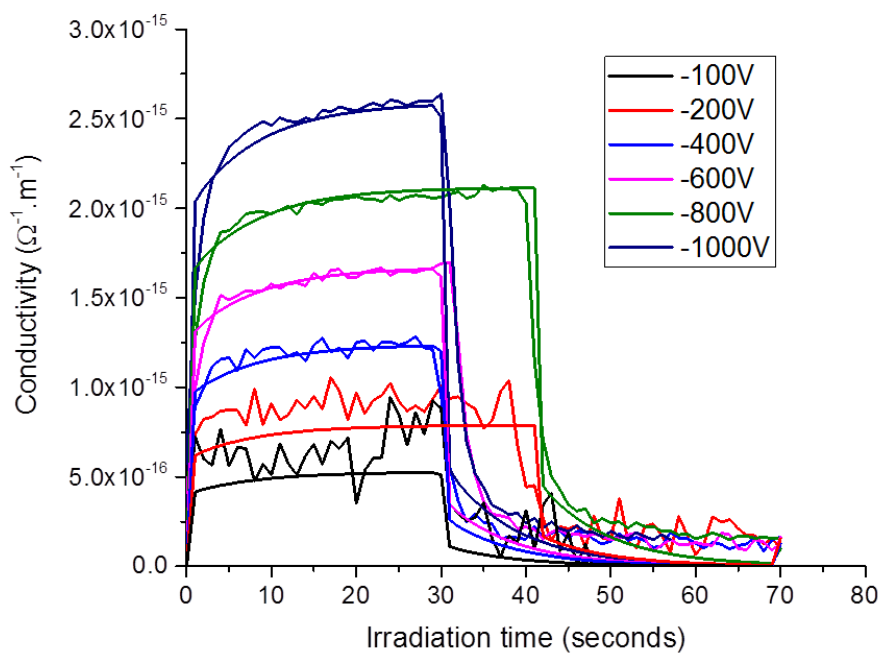

Fig. 12. Results are those from fig. 5. Fits are made using the theory related to both the electric field assisted de-trapping phenomena and the Onsager process.

Like in fig. 9, fig. 13 compares the normalized data of conductivities with the relation $\exp (\beta \sqrt{E})$ that describes the electric field assisted de-trapping processes. Data were normalized with the electric field $E=1,6 \times 10^{7} \mathrm{~V} / \mathrm{m}$. We tried to fit the experimental data from fig. 6 and fig. 7 with the following $\beta$ coefficients: $\beta=1 \times 10^{-4} \mathrm{~m}^{1 / 2} V^{-1 / 2}, \beta=3 \times$ $10^{-4} \mathrm{~m}^{1 / 2} V^{-1 / 2}$ and $\beta=5 \times 10^{-4} \mathrm{~m}^{1 / 2} V^{-1 / 2}$. The best fit corresponds to $\beta=3 \times 10^{-4} \mathrm{~m}^{1 / 2} V^{-1 / 2}$. It has been observed that the electric field assisted de-trapping mechanisms are able to reproduce the experimental measurements for low and high 
electric fields. It was not the case for Onsager since the theory was in good agreement with only low electric fields. As stated before, it suggests that the Onsager mechanism is quite weak for Kapton-HN and that the electric field assisted de-trapping processes are responsible for the conductivity behaviors.

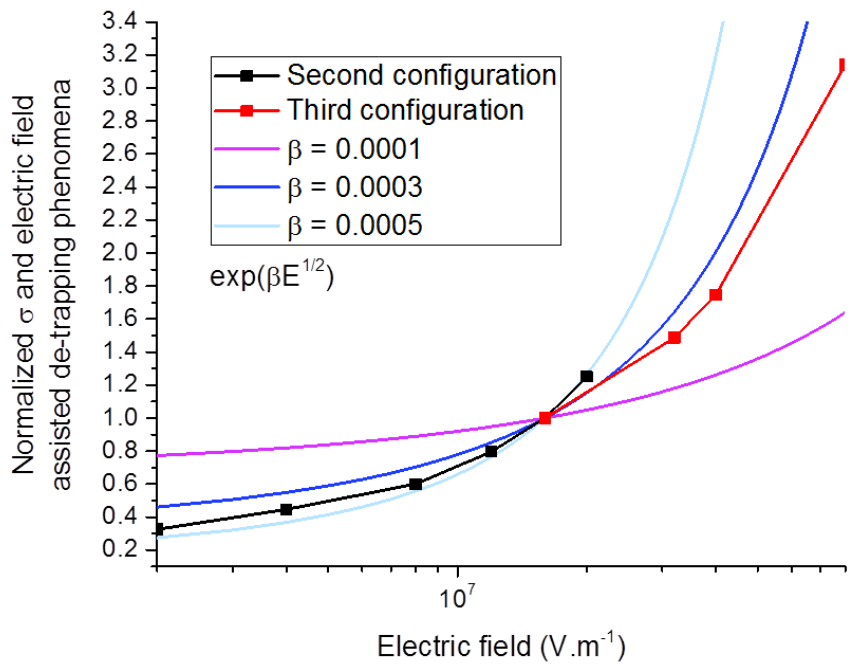

Fig. 13. Comparison between the measured conductivities from fig. 6 and fig. 7 with the electric field assisted de-trapping processes and three different coefficients: $\quad \beta=1 \times 10^{-4} \mathrm{~m}^{1 / 2} V^{-1 / 2}, \quad \beta=3 \times 10^{-4} \mathrm{~m}^{1 / 2} V^{-1 / 2} \quad$ and $\beta=5 \times 10^{-4} \mathrm{~m}^{1 / 2} V^{-1 / 2}$.

\section{ELECTRIC FIELD ASSISTED DE-TRAPPING PROCESSES RELATED TO DEEP TRAPS}

The previous section strongly suggests that the predominant processes for describing the effects of the electric field on the RIC in Kapton-HN are the electric field assisted de-trapping mechanisms related to shallow traps. In this section we extend our analysis to the electric field assisted de-trapping mechanisms related to deep traps. For deep traps, direct tunneling and hopping transport are unlikely. As a result, the de-trapping rate of charges from deep traps is only related to the Poole-Frenkel mechanism. Besides, the influence of the electric field on the de-trapping rate of charges from deep traps is only significant for holes. Previous experiments led at ONERA showed that the electron de-trapping time is very long compared to holes. Once electrons are trapped in the Fermi band they stay in that trapping configuration for a long time. Even when they undergo high electric fields, they remain trapped and cannot reach the conduction band. This phenomenon has not been observed for holes. It means that high electric fields have an influence on hole de-trapping characteristic time as follows (Poole-Frenkel theory):

$\frac{1}{\tau_{p_{t}}}=\frac{1}{\tau_{p_{t 0}}} \times \exp \left(\frac{-e E_{a}+\beta_{P F} \sqrt{E} k_{B} T}{k_{B} T}\right)$

$\beta_{P F}=\frac{\left(\frac{e^{3}}{\pi \varepsilon_{r} \varepsilon_{0}}\right)^{1 / 2}}{k T}$

Where $\tau_{p_{t 0}}$ is a constant. $E_{a}(e V)$ is the activation energy for holes. It corresponds to the energetic level of the trap referenced to the top of the valence band. Under the influence of an electric field the activation energy is lowered by a PooleFrenkel factor.

Fig. 14 displays the experimental and numerical surface potential corresponding to the $l^{\text {st }}$ configuration. Fig. 14 displays 3 curves. The black curve corresponds to experimental data. The blue curve corresponds to numerical results when both the Onsager mechanism and the electric field assisted de-trapping processes (for shallow traps) are taken into account. The red curve gathers all mechanisms: Onsager and the electric field assisted de-trapping processes for shallow and deep traps (Poole-Frenkel). Fig. 14 shows three phases for the surface potential of Kapton-HN as a function of irradiation. In the $1^{\text {st }}$ phase (until 1200 seconds after irradiation began) the surface potential quickly increases. In the $2^{\text {nd }}$ phase (from 1200 seconds after irradiation began) it slowly decreases. In the $3^{\text {rd }}$ phase it quickly drops due to irradiation shut-down. In the $1^{\text {st }}$ phase incident electrons get quickly trapped in the material bulk. Moreover, the generation of free electrons and free holes due to dose deposition by incident electrons quickly leads to the trapping of these charges. As a result, implanted electrons and trapped charges increase the surface potential (and therefore the electric field) of Kapton-HN. In the $2^{\text {nd }}$ phase the electric field due to bulk space charge is high enough so that electric field assisted detrapping (shallow and deep traps) processes occur. The detrapping rate of trapped holes slowly increases which increases the number of free holes. It thus leads to an increase of the bulk conductivity and to a decrease of the surface potential. In the $3^{\text {rd }}$ phase the irradiation is stopped which means there are no more incident electrons that get implanted in the material bulk: the surface potential drops. Under the influence of the electric field due to bulk space charge, trapped holes are released into the Valence band and become free. These free holes move to the material surface which results in its surface potential decrease.

The effect of the electric field on hole de-trapping characteristic time is more noticeable during the relaxation phase because there is no generation of free charges to hide its effect. We have better agreement with the experimental results when we take into account equation (16) in our model. Subsequently, this strongly suggests that the RIC in Kapton$\mathrm{HN}$ is steered by the electric field assisted de-trapping processes related to shallow and deep traps. 


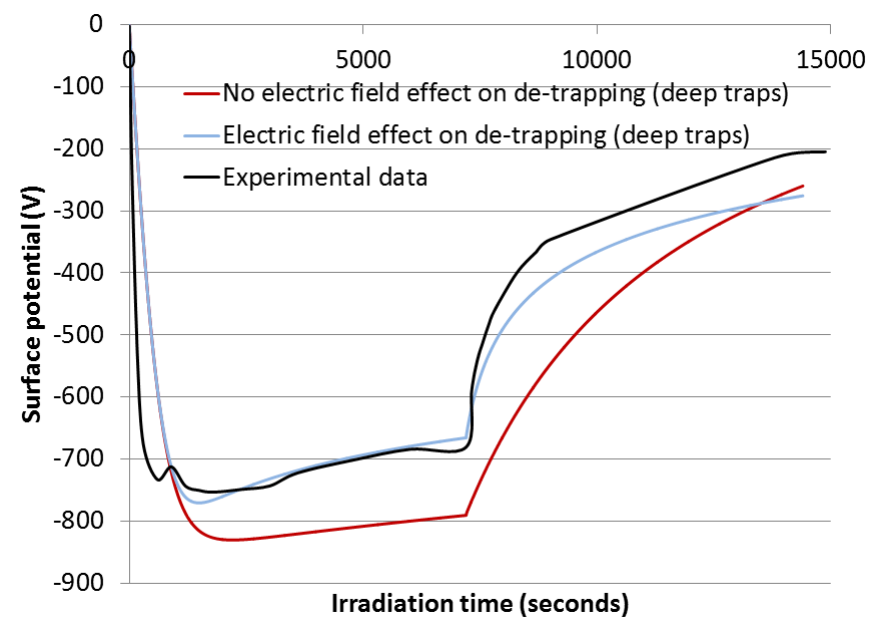

Fig. 14. Experimental and numerical evolution of the surface potential for a Kapton-HN sample of $50 \mu \mathrm{m}$ under the SIRENE spectrum. The sample is irradiated for 2 hours and relaxed for another 3 hours.

\section{CONCLUSION}

In this paper the development of a 1D conductivity model for the description of charge transport in dielectric materials has been presented. Our study focused on Kapton-HN. In a previous paper we demonstrated that the 1-D model gives a good description of charge transport. However, it presented some limitations as we did not take into account the effect of the electric field on de-trapping processes. The influence of the electric field on charge transport was therefore not fully described. This influence was brought into evidence through experiments described in this paper. Several authors introduced the Onsager theory, direct tunneling, hopping transport and the Poole-Frenkel theory as a way to explain the electric field dependence on conductivity. Therefore, we further looked into these mechanisms. At room temperature $(T=300 K)$ and given the range of the electric fields $(2 \times$ $\left.10^{6} \mathrm{~V} / \mathrm{m}<E<8 \times 10^{7} \mathrm{~V} / \mathrm{m}\right)$, it seems that the Onsager process can be discarded for Kapton-HN in these conditions. It does not have much influence on conductivity. On the other hand, electric field assisted de-trapping (shallow and deep traps) processes can account for the conductivity dependence with the electric field. The influence of the electric field on the electron and hole mean mobility and on the de-trapping rate of deep trapped holes was analyzed and yielded good results: numerical conductivity and numerical surface potential profiles fit the experimental data very well.

Great progress has been done with the 1D model. The next step is to take into account the effect of temperature on conductivity. This effect was brought into evidence through different experiments performed at ONERA. We discarded here the electric field influence of the Onsager theory on conductivity at room temperature for Kapton-HN. However, it might not be the case at different temperature levels. Further analysis will be carried out on Onsager and electric field assisted de-trapping processes in order to understand the influence of temperature on conductivity and surface potential levels.

\section{ACKNOWLEDGEMENTS}

The authors would thank the region Occitanie and ONERA for their $\mathrm{PhD}$ funding for Rémi Pacaud. This work has been performed in part in collaboration and with support of the CNES R\&T program to develop this 1D model and compare it with experimental results.

\section{REFERENCES}

[1] R. Hanna et al., "Radiation induced conductivity in Teflon FEP irradiated with multienergetic electron beam", IEEE Trans. Plasma Sci., vol. 41, no. 12, pp. 3520-3525, Dec. 2013.

[2] R. Hanna et al., "Radiation Induced conductivity in space dielectric materials," J. Appl. Phys., vol. 115, no. 3, p. 033713, 2014.

[3] T. Paulmier, B. Dirassen, M. Arnaout, D. Payan, and N. Balcon, "Radiation-induced conductivity of space used polymers under high energy electron irradiation," IEEE Trans. Plasma Sci., vol. 43, no. 9, pp. 2907-2914, Sep. 2015.

[4] R. Pacaud, T. Paulmier, P. Sarrailh, "1-D Physical Model of Charge Distribution and Transport in Dielectric Materials Under Space Radiations," IEEE Trans. Plasma Sci., vol. 45, no. 8, pp. 1947-1954, Aug. 2017.

[5] T. Paulmier, B. Dirassen, D. Payan, and M. Arnaout, "Analysis of Charge Transport and Ionization Effect in Space-Used Polymers Under High-Energy Electron Irradiation," IEEE Trans. Plasma Sci., vol. 45, no. 8, pp. 1933-1937, Aug. 2017.

[6] J. Boch, F. Saigne, L. Dusseau, and R. D. Schrimpf, "Temperature effect on geminate recombination," Appl. Phys. Lett., vol. 89, no. 4, p. 042108, 2006.

[7] A. P. Tyutnev et al, "Frequency factor of the semiempirical model for the radiation-induced conductivity in spacecraft polymers," in Proc. 14th Spacecraft Charging Technol. Conf. (SCTC), Noordwijk, NL, Apr. 2016.

[8] S. D. Ganichev, E. Ziemann, W. Prettl, I.N. Yassievich, A.A. Istratov, E.R. Weber, "Distinction between the Poole-Frenkel and tunneling models of electric-field stimulated carrier emission from deep levels in semiconductors," Phys. Rev. B 61 (2000) 10361-10365.

[9] Maneesha Garg, J. K Quamara, Nucl. Instr. And Meth. B 179 (2001) 83.

[10] P. A. Martin, B. G. Streetman, and K. Hess, "Electric field enhanced emission from nonCoulombic traps in semiconductors," J. Appl. Phys., vol. 52, no. 12, 7409 (1981).

[11] T. Paulmier et al, "Experimental test facilities for representative characterization of space used materials," in Proc. 13th Spacecraft Charging Technol. Conf. (SCTC), Pasadena, CA, USA, Jun. 2014.

[12] R. Hanna, "Transport de charges et mécanismes de relaxation dans les matériaux diélectriques à usage spatial," Ph.D. dissertation, Dept. Génie Elect., Univ. Toulouse, Toulouse, France, 2012.

[13] Tyutnev, A.P., Nikerov, A.V., Abrameshin, A.E. et al. Polym. Sci. Ser. A (2014) 56: 719. 